Refereed version published in Electroanalysis 2010, 22, No. 24, 2961 - 2966

\title{
Electrochemical behaviour of carbamazepine in acetonitrile and dimethylformamide using glassy carbon electrodes and microelectrodes
}

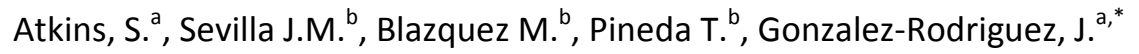

(a) School of Natural and Applied Sciences, University of Lincoln, Brayford Pool, Lincoln, LN6 7TS, United Kingdom

(b) Departamento de Quimica Fisica y Termodinamica Aplicada, Universidad de Cordoba, Campus de Rabanales, Ed. Marie Curie, E-14071 Cordoba, Spain

$(*)$ corresponding author

\begin{abstract}
The electrochemical reduction of carbamazepine in acetonitrile (ACN) and dimethylformamide (DMF) using a glassy carbon electrode and microelectrodes has been studied. The reduction process is consistent with an Electrochemical-Chemical mechanism (EC) involving a two electron transfer followed by a first order reaction, as shown by the cyclic voltammetry and differential pulse voltammetry. Halfwave potential, number of electron transfer, diffusion coefficient and rate constant of the associated chemical reaction are reported. Detection limits are in DPV: LoD $=0.92$ and $0.76 \mu \mathrm{gL}^{-1}$ in $A C N$ and DMF, respectively. Precision (\%RSD) and recovery (\%) values when pharmaceutical compounds (200mg carbamazepine tablets) and spiked plasma samples were tested ranged from 1.09 to $9.04 \%$ and $\%$ recoveries ranged from 96 to $104.1 \%$.
\end{abstract}

Keywords: carbamazepine, glassy carbon electrode, microelectrodes, voltammetry 


\section{Introduction}

Carbamazepine (CBZ) is an antiepileptic drug used for some conditions, such as epilepsy and bipolar disorder. It is usually administered orally in tablet or suspension form presenting $85 \%$ compound bioavailability. It is excreted through urine in a $72 \%$ (1-3\% as unchanged drug) and in a $28 \%$ through faeces [1].

The drug presents high capacity for interaction by enhancing or reducing the metabolism of other drugs. There are multiple evidences demonstrating the potential for the misuse of carbamazepine. The drug combined with alcohol has been previously reported to be abused [2] producing euphoria comparable to that obtained when combining benzodiazepines and alcohol as it stimulates the activation of dopaminergic and serotonergic systems. Carbamazepine has also been linked to cases of sexual assault $[3,4]$ in which alcohol and other drugs were also involved and in cases of suicide or misuse of drugs in which combination with other licit or illicit drugs resulted in death [5]. It is not strange to find it in many cases in which drug interaction has also been the cause of fatality. Jönsson et al [6] reported CBZ to be the $16^{\text {th }}$ commonest drug out of 50 found in post-mortem samples from deceased drug addicts in Sweden. Cases in which the drug has also be found to be the main cause of death by overdose and not associated to interaction with other drugs are also frequent $[7,8]$.

The drug is usually analysed in biological samples, such as plasma or urine, using liquid chromatographic methods. Liquid chromatographic methods using both UV-Vis [9] and mass spectrometry detectors [10] are the most common techniques of choice by forensic laboratories and they offer the highest selectivity and sensitivity in its detection. Biological samples need to be cleaned-up prior to analysis, which can be time consuming. Different analytical techniques have been used for this purpose, including dispersive liquid-liquid extraction [9], solid phase extraction [10] and solid phase microextraction [11].

Electrochemical methods for the analysis of carbamazepine are scarce. Moses et al first described the behaviour of carbamazepine when analysed using mercury electrodes [12] and more recently GarciaGarcia et al described the determination of carbamazepine in pharmaceutical solutions using differential-pulse adsorptive stripping Voltammetry and the oxidation properties of the molecule with different modified and unmodified carbon electrodes [13].

The potential for the direct use in the determination of carbamazepine in different matrices, both in tablets and biological samples, is still an area that needs exploring. The work presented here presents the analytical characteristics and electrochemical reduction mechanism of a carbamazepine method based on the reduction of the molecule on glassy carbon electrodes and microelectrodes. To the best of our knowledge this is the first time that this reduction mechanism has been reported for these types of electrode. Our study offers an alternative to other analytical methods, as these solid electrodes present analytical characteristics that made them suitable for cheap and fast analysis. The work on glassy carbon electrodes and microelectrodes opens the door for economic, miniaturised devices suitable for the fast determination of carbamazepine in different matrices within the forensic and pharmaceutical fields. 


\section{Experimental}

Carbamazepine (CBZ) (99\% purity), tetra-butyl ammonium perchlorate (TBAP) (98\% purity) and Lyophilised human plasma were purchased from Sigma-Aldrich (Darmstadt, Germany). Ethanol, Acetonitrile (ACN) and Dimethylformamide (DMF) (HPLC grade) were also purchased from SigmaAldrich. Tegretol tablets containing $200 \mathrm{mg}$ of carbamazepine were purchased from Novartis (Varese, Italy).

Cyclic Voltammetry (CV) and Differential Pulse Voltammetry (DPV) analyses were performed with a CHI $900 \mathrm{~b}$ instrument (manufacturer, city) attached to a PC fitted with control software for data acquisition. DPV conditions for all experiments were: pulse amplitude $0.05 \mathrm{~V}$, pulse width $0.05 \mathrm{~s}$ and pulse period 0.2 s. Experimental optimisation and experimental parameters were calculated using DigiSim 3.03 software. A three electrode configuration was used: graphite electrodes acting as working electrodes, SCE was used as a reference electrode and a platinum electrode acted as a counter electrode. The working electrodes used for the different experiments were either a $3 \mathrm{~mm}$ diameter glassy carbon electrode or a graphite $11 \mu \mathrm{m}$ diameter micro disk electrode from Bioanalytical Systems (Kenilworth, UK). The working electrodes were polished with $0.05 \mu \mathrm{m}$ slurry, rinsed and sonicated in $18 \mathrm{M} \Omega$ Milli-Q water (Millipore, Mariland, US) and dried before use. Nitrogen was bubbled in the electrochemical cell for $20 \mathrm{~min}$ before analysis.

\section{Results and Discussion}

\subsection{Cyclic Voltammetry (CV)}

Figure 1 shows the different voltammograms obtained by Cyclic Voltammetry (CV) corresponding to the electrochemical reduction of a $1 \mathrm{mM}$ carbamazepine (CBZ) solution using a $3 \mathrm{~mm}$ glassy carbon electrode at different sweep rates. The solvent used in this experiment was acetonitrile and the supporting electrolyte was $0.1 \mathrm{M}$ TBAP. Reversible waves and a decrease in the anodic current can be observed with increasing and decreasing sweep rates, respectively. This behaviour in CV is consistent with an EC mechanism [14], which implies that a chemical reaction is coupled to the electrochemical process to produce a non-electro active product. As a consequence of this secondary process, the electroactive substance produced in the reduction of CBZ is removed from the surface of the electrode and therefore the associated oxidation current disappears.

In the insert, in Figure 1, we also find more evidences in support of this mechanism. In this case the sweep rate was kept constant and the solvent used was dimethylformamide, which presents a very similar behaviour to that exhibited by ACN. The switching potential $\left(E_{\lambda}\right)$ was changed in this experiment to more cathodic potentials in each analysis. It is clear from the figure that the oxidation peak diminished when more negative cathodic potentials were used as switch potential. As the difference between the potential at which we obtain the cathodic peak $\left(E_{p}{ }^{c}\right)$ and the switch potential $\left(E_{\lambda}\right)$ increases so does the time, allowing the chemical reaction coupled to the electrodic process to progress further. This will remove more of the electro active substance from the surface of the electrode, thus reducing 
the oxidation peak. This supports the idea of a EC mechanisms associated to the reduction of CBZ. This mechanism can be described as follows:

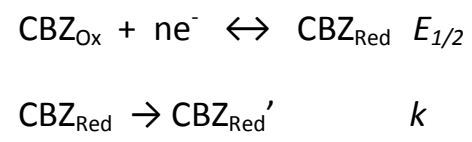

Moses et al. [12] studied the electrochemical behaviour of CBZ in aqueous solutions using mercury electrodes, in which they found a bi-electronic reduction mechanism. This mechanism implies the saturation of the 10,11 double bond present in the heterocycle of the molecule. They also suggested an EC mechanism to explain the behaviour in mercury and suggested that the associated chemical reaction could be a conformational isomerisation to yield a non-electro active. Our results in non protonated solvents support this idea and supporting this mechanism. A remarkable difference in the behaviour of $\mathrm{CBZ}$ in non protonated solvents and using a glassy carbon electrode is the shift in the reduction potential of the molecule. Moses et al. reported a reduction potential of $-1.64 \mathrm{~V}$. vs SCE whereas in the conditions described for our experiments the reduction potential is shifted towards more cathodic potential, over $-2 \mathrm{~V}$ (Fig. 1).

Table 1 shows a summary of the most relevant results obtained with CV for both solvents (both ACN and DMF). These results clearly show that the reduction of CBZ is diffusion controlled. It also demonstrates that the electron transfer process is reversible in the sweep rate range specified and it also shows an important specific influence of the solvent in the electron transfer. Out of this range $E_{p}^{c}$ is shifted towards more negative potentials. This is explained because a decrease in sweep rate will increase the effect of the chemical reaction in the electrochemical process in the zone for kinetic behaviour. On the contrary, an increase in the sweep rate will influence the electrodic process, as expected, indicating a quasi-reversible profile for the process. Some effect of non compensated solution resistance is not discarded at the larger scan rate.

Once the mechanism was elucidated we proceed to calculate the diffusion coefficient (D) of CBZ for the two solvents studied. In order to do this we simulated the process using DigiSim 3.0 at high sweep rate, in which the effect of the coupled chemical reaction is negligible and the process is reversible. We obtained two values of $(7.29 \pm 0.45) \times 10^{-6}$ and $(4.78 \pm 0.31) \times 10^{-6} \mathrm{~cm}^{2} / \mathrm{s}$ for both $A C N$ and DMF, respectively. These values are in good agreement with the viscosity relationship between the two solvents ( $D$ decreases with an increase in viscosity) and with similar tri-cyclic components found in the literature in organic solvents $[15,16]$.

From the concentration studies on the response of CBZ using a sweep rate off the kinetic limits for the EC process $(v>50 \mathrm{mV} / \mathrm{s})$ can be observed that $i_{p}^{c}$ is proportional to concentration for both solvents and $E_{p}^{c}$ is constant. In the kinetic range $(v=20 \mathrm{mV} / \mathrm{s})$ this behaviour is very similar, which demonstrates that the chemical reaction coupled to the electrodic process is not a second order reaction depending on the $\mathrm{CBZ}_{\mathrm{R}}$ concentration. This also seems to support the assumption that the reaction observed in nonprotonated solvents is of the same nature than that observed in aqueous solution. 
Figure 2 shows the relationship between $i_{a} / i_{c}$ and the sweep rate. It can be observed that the ratio between the anodic and cathodic intensities increases with the sweep rate in both solvents, as it was already observed in Figure 1 and backing the existence of an EC process. It can also be observed that the intensity ratios obtained for $A C N$ are lower than those obtained for DMF. This suggests that the rate constant for the coupled chemical reaction must be higher in ACN. The obtained curves for this electrochemical reaction scheme [17] provides with values for the rate constant consistent with a first order reaction kinetic scheme. The values obtained for the rate constants were $0.177 \pm 0.017 \mathrm{~s}^{-1}$ for DMF and $0.280 \pm 0.011 \mathrm{~s}^{-1}$ for ACN. These values are lower that those obtained from the literature for a similar process in aqueous solutions [12].

From this study with concentration we can also determine the limit of detection for CBZ in these solvents. The results obtained were $3 \mu \mathrm{gL}^{-1}$ for ACN and $1 \mu \mathrm{g} \mathrm{mL}^{-1}$ for DMF. These results can be explained based on a higher background current observed in the system ACN/TBAP when compared to that obtained when using the DMF/TBAP system (even taking into account that DMF presents higher viscosity and therefore lower faradic current tend to be observed). Calibration curves also showed good linearity $\left(r^{2}=A C N: 0.9973, D M F: 0.9970\right)$

\subsection{Differential Pulse Voltammetry (DPV)}

Figure 3 shows the voltammograms obtained for CBZ using DPV under the same experimental conditions that those used in CV. The cathodic potentials obtained were $-2.25 \mathrm{~V}$ and $-2.15 \mathrm{~V}$ for ACN and DMF, respectively. This shows good agreement with the values obtained for $E_{1 / 2}$ in $\mathrm{CV}$ (table 1 ).

For both solvents, it can be observed that the anodic peak current value is higher than that of the anodic peak. This behaviour suggests that the electrochemical process it is not fully reversible (Nerstian processes). Complex electrochemical processes [18] can be identified by comparing variables, such as the ratio of intensities of the anodic and cathodic peaks $\left(i_{a} / i_{c}\right)$, the difference between peak potentials $\left(E_{p}^{c}-E_{p}^{a}\right)$ and the half-peak width ratio $\left(w_{1 / 2}^{a} / W_{1 / 2}^{c}\right)$. These were calculated for ACN and DMF, being $0.77,30 \mathrm{mV}, 1.02$ and $0.55,39 \mathrm{mV}$ and 1.07 , respectively. In both cases they lay within the values characteristic for an EC process being the chemical stage a first order reaction.

Figure 4 shows cathodic DPV curves obtained when CBZ is analysed in ACN in an interval of concentrations ranging from 0.02 to $0.2 \mathrm{mg} / \mathrm{mL}$. The reduction potential of the cathodic sweep is independent of $C B Z$ concentration with a value of $-2.22 \mathrm{~V}$. The signal obtained increases linearly with $C B Z$ concentration with a correlation coefficient of $r^{2}=0.9994$ for the line of best fit in ACN and $r^{2}=0.9975$. A similar behaviour is obtained when DMF was used as solvent. The limits of detection obtained for both ACN and DMF were 0.92 and $0.76 \mu \mathrm{gL}^{-1}$ respectively. These results are in line with the expectations as Differential Pulse Voltammetry always presents a much better sensitivity than Cyclic Voltammetry.

These limits of detection allow detection of the drug in tablets, analysis of therapeutic levels of CBZ in plasma (4-12 $\mathrm{g} \mathrm{mLL}^{-1}$ ) or even potentially the concentration of non-metabolised CBZ in urine [1] (after pre-concentration). Four tablets and four samples of human plasma spiked with carbamazepine were 
analysed using DPV. Tegretol tablets (CBZ 200mg) were crushed and dissolved and sonicated in $10 \mathrm{~mL}$ $A C N$. Plasma samples were spiked using an ethanolic solution of $C B Z$ to obtain a final concentration of $10 \mu \mathrm{g} \mathrm{mL}^{-1}$. The results obtained are summarised table 2 .

\subsection{Study with microelectrodes}

Carbamazepine was also studied using a graphite micro disc electrode of nominal diameter of $11 \mu \mathrm{m}$ and the intensity vs potential curves recorded under the same experimental conditions described before. Figure 5 shows the plots obtained for CBZ together with those obtained for ferrocenemethanol in water. The latter has been used as reference as it can be considered a model as it is well known that the molecule undergo oxidation through a reversible mono-electronic process and the diffusion coefficient is also known [19]. Data obtained with the microelectrode for CBZ agree with those previously obtained with glassy carbon. Thus the half wave potential obtained for the microelectrode is 2.20 and $-2.14 \mathrm{~V}$, in ACN and DMF respectively. The limiting current obtained for the electronic process is also consistent with a bi-electronic transfer. The diffusion coefficients can also be calculated using the equation:

$i_{L}=4 n F D C a$

where, $n=$ number of electrons, $F=$ Faraday constant, $D=$ diffusion coefficient, $C=$ concentration of the electroactive substance and $a=$ radius of the micro disc electrode

The results obtained were $(6.70 \pm 0.22) \times 10^{-6}$ and $(4.64 \pm 0.22) \times 10^{-6} \mathrm{~cm}^{2} \mathrm{~s}^{-1}$ in ACN and DMF, respectively, showing good agreement with those obtained using a glassy carbon electrode by CV. Results for different CBZ concentrations were also obtained in the range 0.2 to $1 \mathrm{mM}$ using DPV. The inset figure in figure 5 shows an increase of the current intensity with increasing CBZ concentration in DMF (linear $r^{2}=$ 0.9996). The limits of detection obtained in $A C N$ and DMF were 2.42 and $2.00 \mu \mathrm{g} \mathrm{mL}^{-1}$, respectively. This increase in the LOD can be explained due to an increase of the background current with increasing negative potentials. This phenomenon can also be observed in the inset of figure 5 .

\section{Conclusions}

The electrochemical reduction of carbamazepine in acetonitrile (ACN) and dimethylformamide (DMF) using a glassy carbon electrode and graphite micro disk electrode has been achieved. The reduction process is consistent with an Electrochemical-Chemical mechanism (EC) involving a two electron transfer followed by a first order reaction. Typical electrochemical parameters, such as half-wave potential, the number of electron transfer, diffusion coefficient and the rate constant of the associated chemical reaction have been reported for the first time for these electrodes. Analytical characteristics, such as LoD, precision, \% recovery and calibration ranges have been also presented. The analysis in Plasma and tablets demonstrates the applicability of the proposed methodology in real samples. 


\section{Acknowledgements}

We thank the Ministerio de Educación y Ciencia (MEC) (Project CTQ2007-62723/BQU) Junta de Andalucía and University of Córdoba for financial support of this work. We also thank the EC Long Life Programme (Erasmus) for financial support which made possible this collaboration.

\section{References}

[1] The Merck Manuals: carbamazepine (drug information provided by Lexi-Comp). Available at: http://www.merck.com/mmpe/lexicomp/carbamazepine.html [accessed 26/03/2010].

[2] G. Sullivan, S. Davis, Journal of Psychopharmacology 1997, 11(1), 93.

[3] A. Wayne Jones, F.C. Kugelberg, A. Holmgren, J. AhIner, Forensic Sci. Int. 2008, 181, 40.

[4] J.A. Hall, C.B.T. Moore, Journal of Forensic and Legal Medicine 2008, 15, 291.

[5] J.L. Pilgrim, et al., Deaths involving serotonergic drugs, Forensic Sci. Int. 2010 (in press), doi:10.1016/j.forsciint.2010.01.014.

[6] A.K. Jönsson, P. Holmgren, H. Druid, J. AhIner, Forensic Science International 2007, 169, 101.

[7] R.S. Fisher, B. Cysyk, J. Toxicol. Clin.Toxicol. 1988, 26, 477.

[8] H.A. Spiller, R.D. Carlisle, J. Forensic Sci. 2001, 46, 1510.

[9] H.A. Mashayekhi, P. Abroomand-Azar, M. Saber-Tehrani, S. W. Husain, Chromatographia 2010, 71, 5.

[10] M. Subramanian, A.K. Birnbaum, R.P. Remmel, Therapeutic Drug Monitoring 2008, 30(3), 347.

[11] M.D. Cantú, D.R. Toso, C.A. Lacerda, F.M. Lanças, E. Carrilho, M.E.C. Queiroz, Analytical and Bioanalytical Chemistry 2006,2, 256.

[12] G.S. Moses, K.M. Rao, N. Srinivasa Rao, A. Ramachandraiah. J. Indian Chem. Soc. 1995, 72, 333

[13] García-García, M.A., Dominguez-Renedo, O., Alonso-Lomillo, A., Arcos-Martínez, M.J. Sensor Letters 2009, $7(4), 586$

[14] A. J. Bard y L. R. Faulkner, en "Electrochemical Methods. Principles and Applications", Chapter 12, p.496-500, J. Wiley and Sons, New York 2001.

[15] A.M. Farrington, J.M. Slater, Analyst 1997, 122, 593

[16] A.E. Carvalho, G.B. Alcantara, S.M. Oliveira, A.C. Micheletti, N.K. Honda, G. Maia, Electrochim. Acta 2009, 54, 2290 
[17] R.S. Nicholson, I. Shain, Anal. Chem. 196436706

[18] M.-H. Kim, L. Yan, R.L. Birke, M.-Z. Czae, Electroanalysis 2003, 15, 1541

[19] N. Anicet, C. Bourdillon, J. Moiroux, J.-M. Saveant, J. Phys.Chem. B102 (1998) 9844 ; C. Cannes, F. Kanoufi, A.J. Bard, J. Electroanal. Chem. 547 (2003) 83; M.P. Longinotti, H.R. Corti; Electrochem.

Communications 9 (2007) 1444

\section{Figures and legends}

Figure 1. Cyclic voltammetry of CBZ $1 \mathrm{mM}$ in ACN solution containing $0.1 \mathrm{M}$ TBAP at several scan rate: $0.01,0.04,0.1,0.2 \mathrm{~V} / \mathrm{s}$.Inset: Electrochemical response at several switching potential in DMF. The arrows indicate the experimental evolution. Scan rate $0.2 \mathrm{~V} / \mathrm{s}$

Figure 2. Plot of la/lc vs scan rate from measurements of cyclic voltammograms of CBZ in ACN and DMF.

Figure 3. DPV of CBZ 1mM. (a) ACN, (b) DMF.

Figure 4. DPV of CBZ at several concentrations in ACN. From top to bottom: $0.03,0.06,0.09,0.12,0.15$, $0.18 \mathrm{mg} / \mathrm{mL}$. Inset: Plot of the cathodic peak current vs concentration.

Figure 5. i-E curves with graphite microelectrode (11- $\mu \mathrm{m}$ diameter). Cyclic voltammetry: (a) Ferrocenemethanol, (b) CBZ in ACN, (c) CBZ in DMF. C=1mM, v=20mV/s. Inset: DPV voltammograms of CBZ with the concentration: from top to bottom: 0, 0.5, 0.6, 0.7, $0.8 \mathrm{mM}$. 
Figure 1

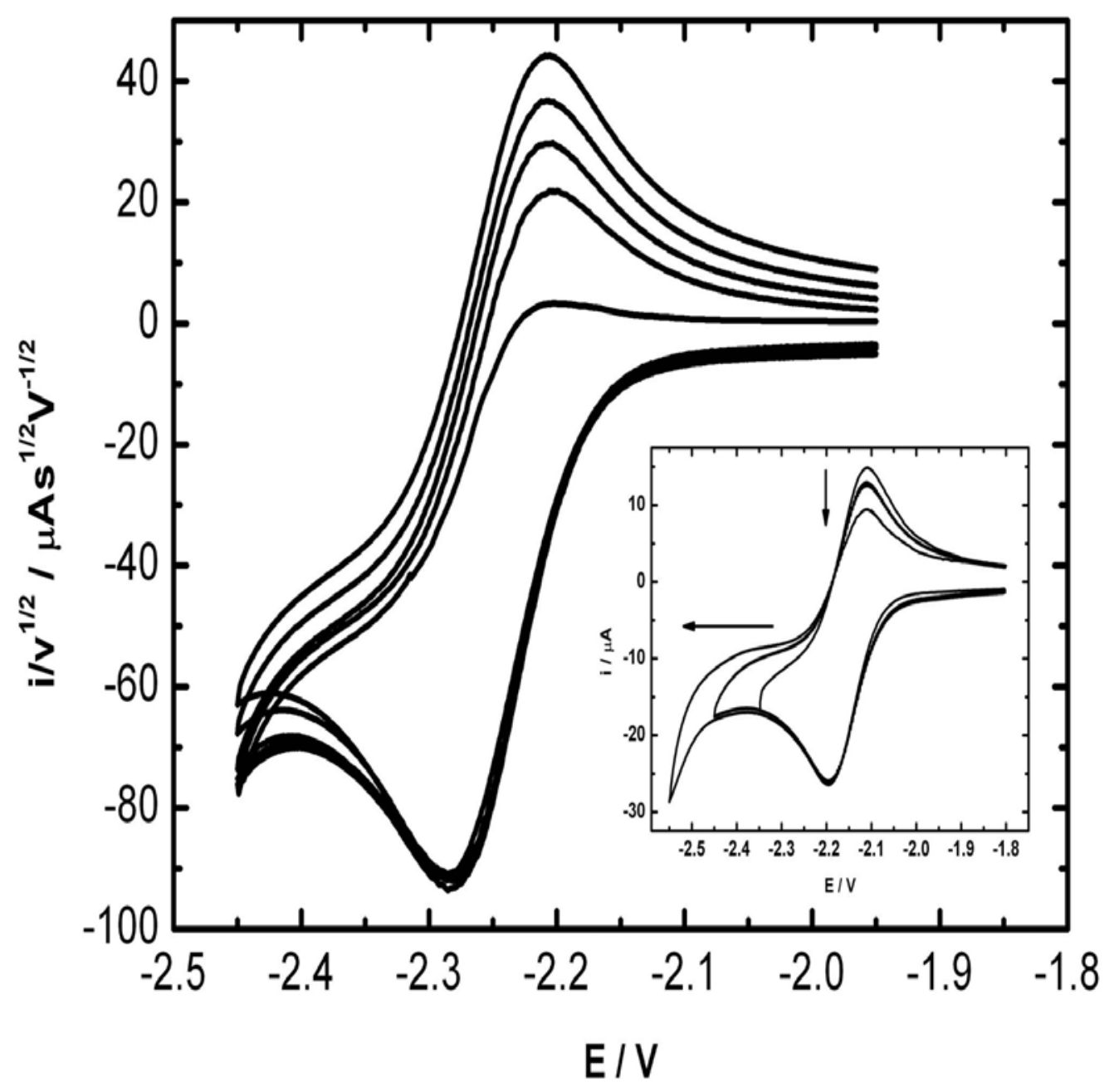


Figure 2

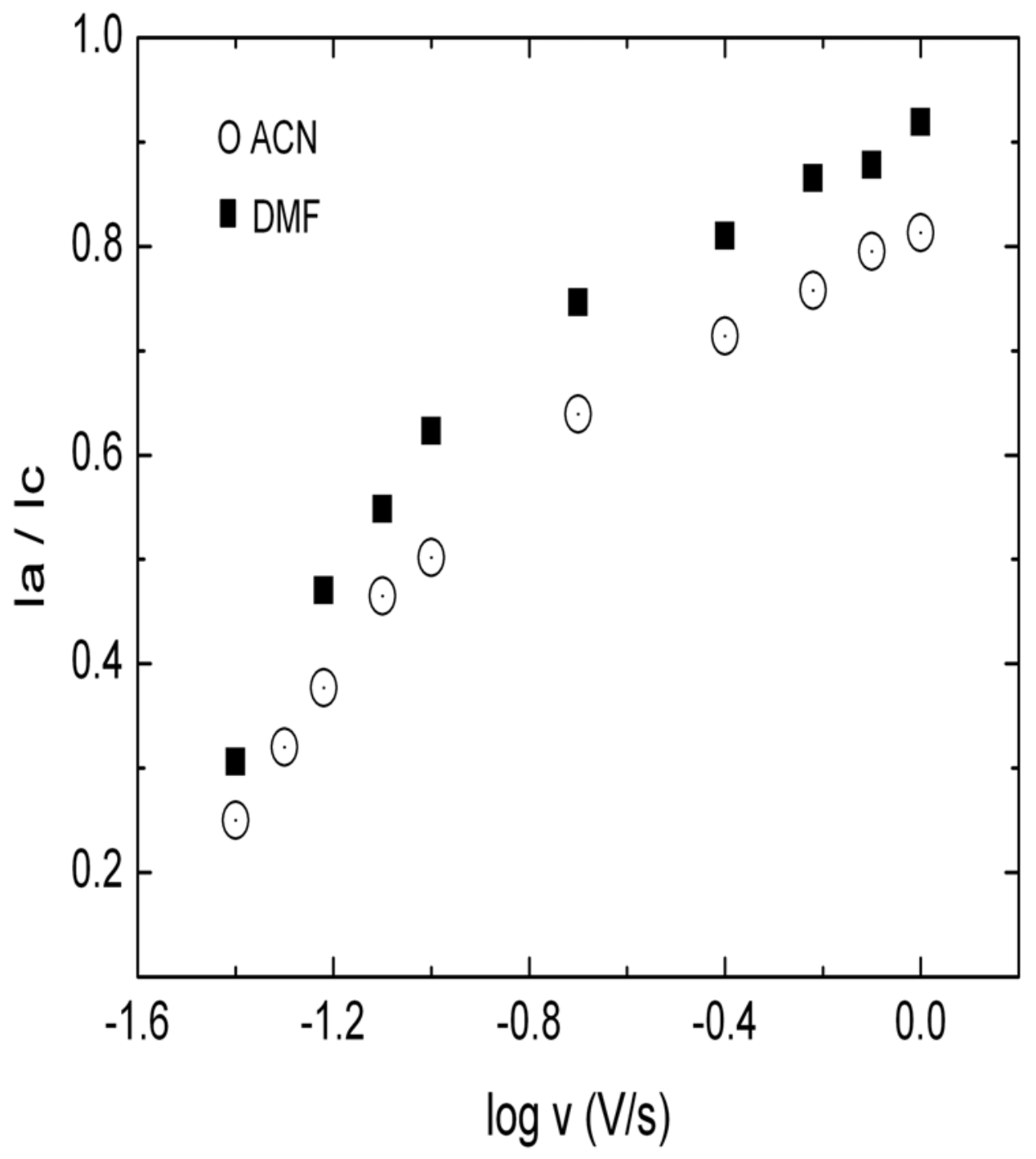


Figure 3

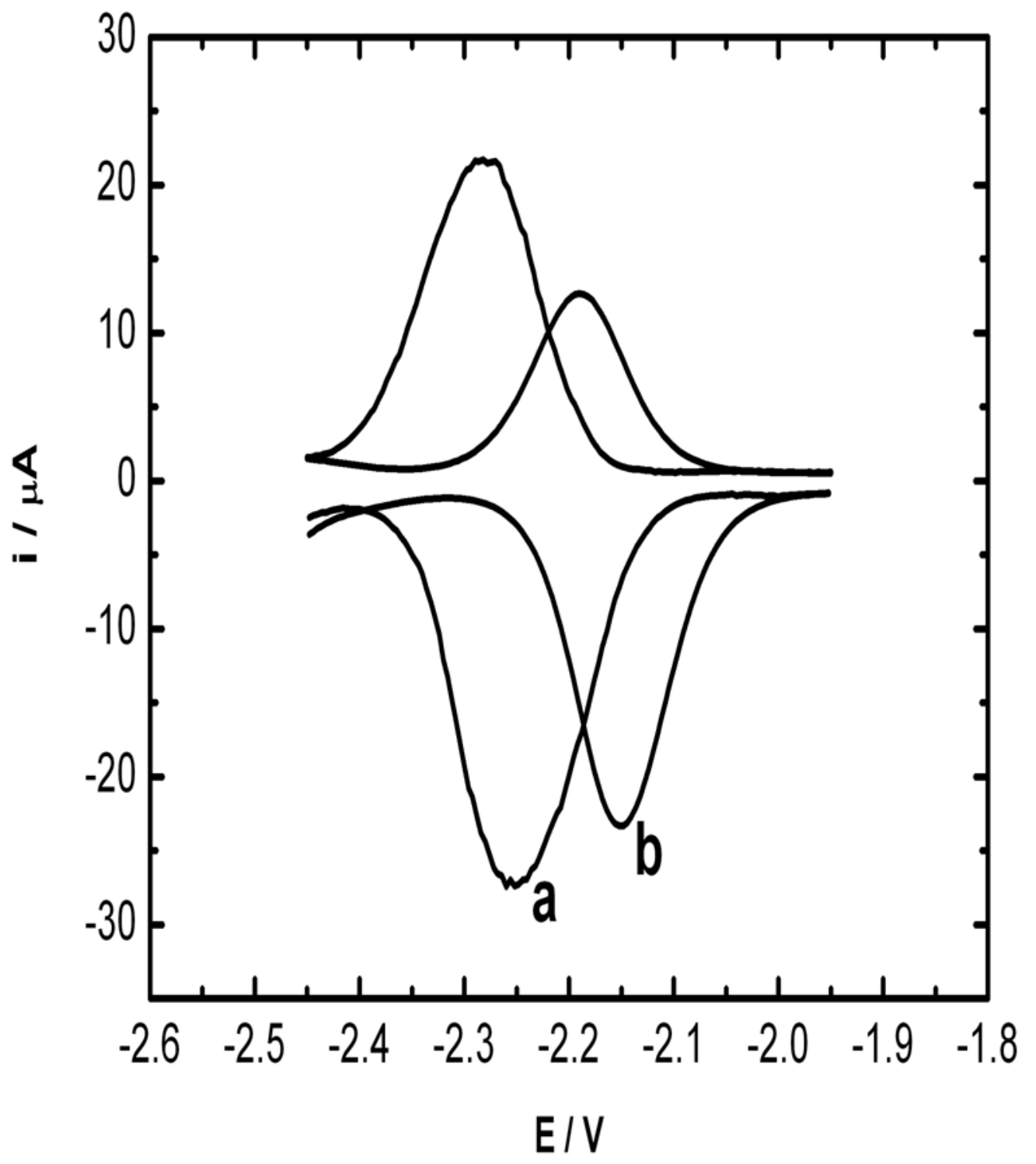


Figure 4

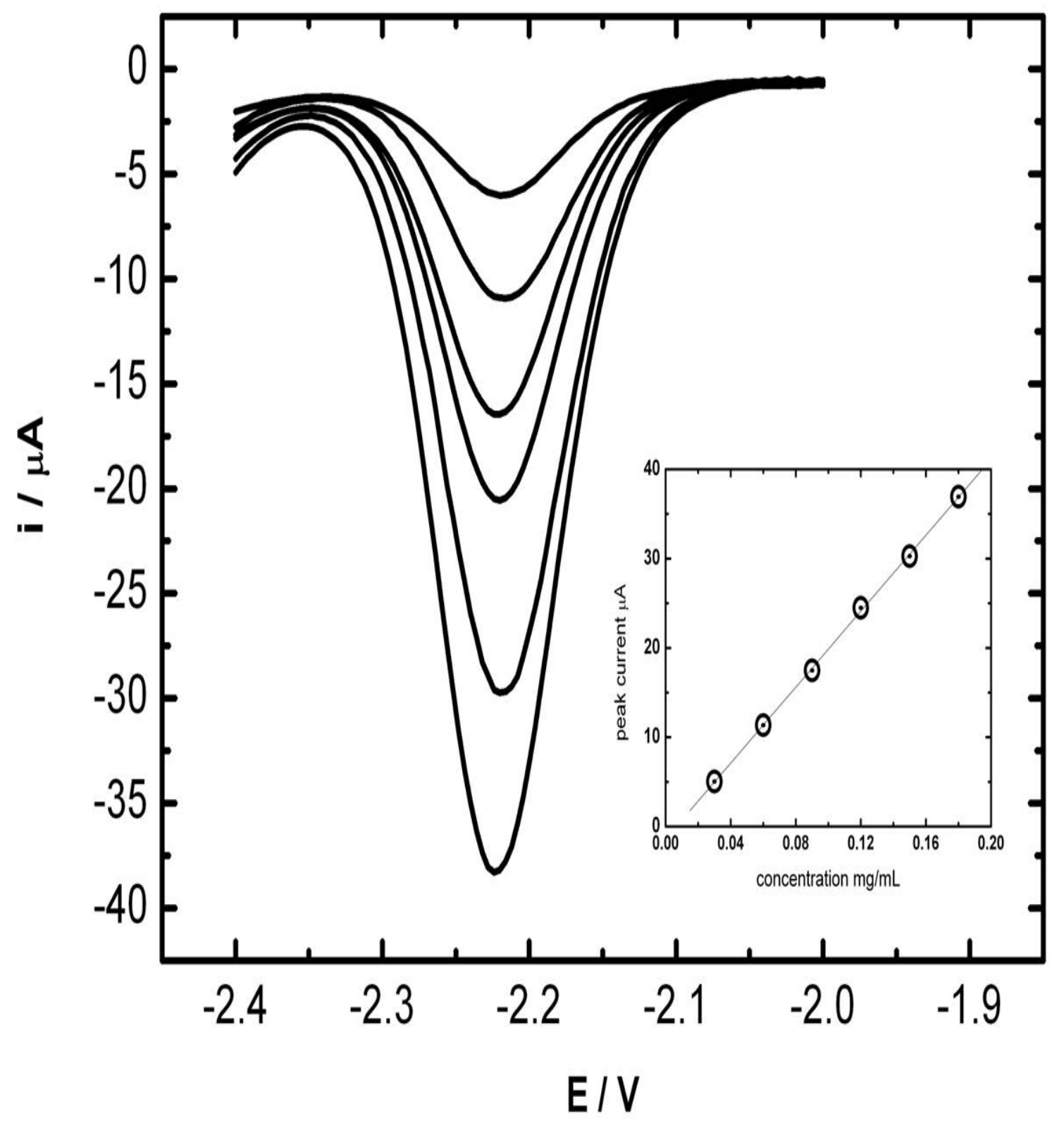


Figure 5

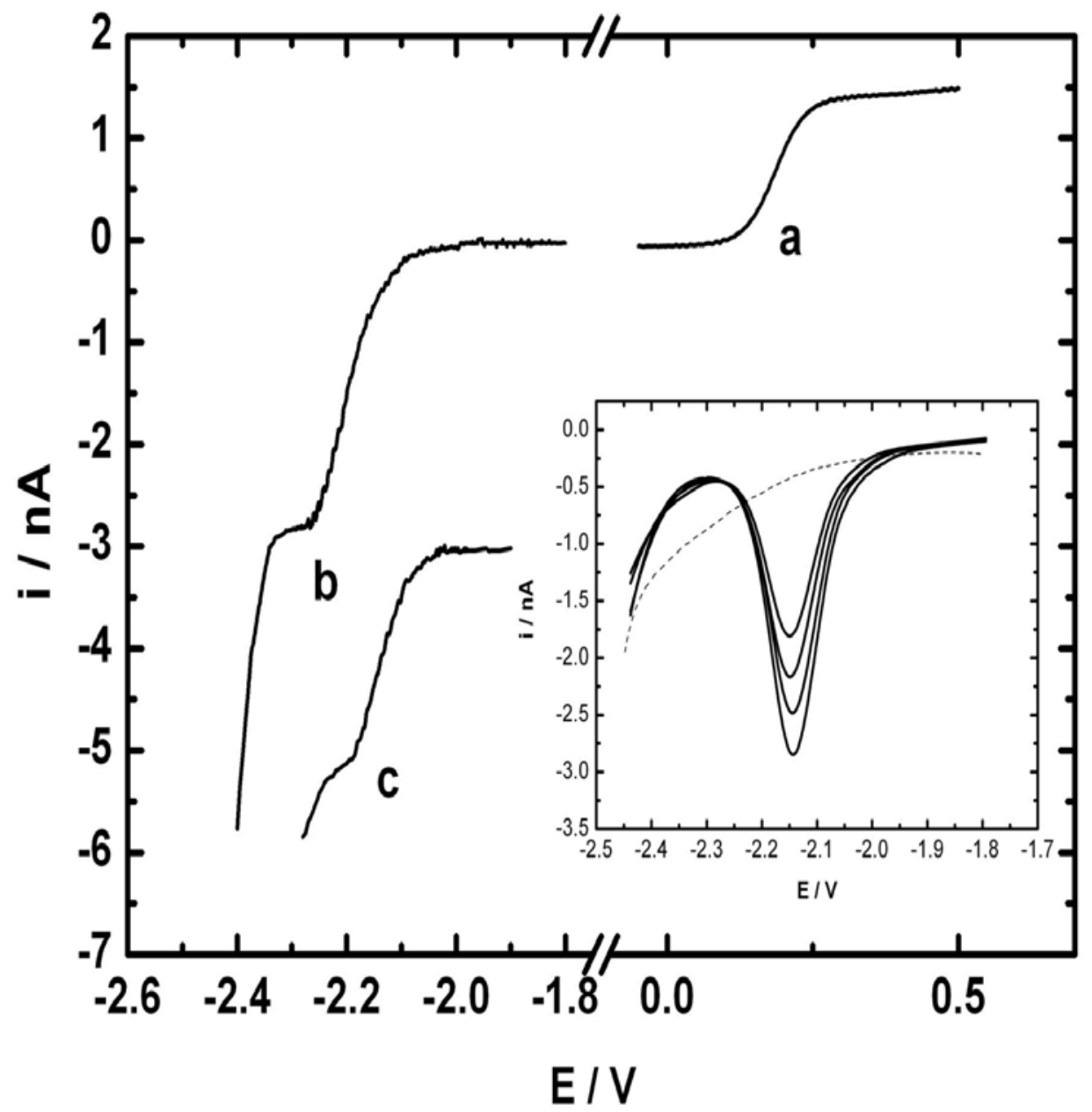


Tables

Table 1. Cyclic voltammetry. CBZ in GC.

\begin{tabular}{|c|c|c|c|c|c|}
\hline & $\log i_{p}$ vs $\log v$ & $E_{1 / 2}(V)$ & $E_{P}^{c} v s \log v$ & $I_{P}^{c}$ vs C & $E_{P}^{c}$ vs c \\
\hline ACN & 0.46 & -2.24 & $\begin{array}{c}\text { Independent } \\
\text { between } 0.05- \\
0.5 \mathrm{Vs}^{-1}\end{array}$ & 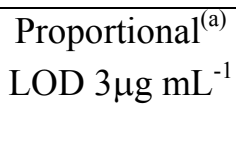 & independent \\
\hline$\overline{\text { DMF }}$ & 0.55 & -2.16 & $\begin{array}{c}\text { Independent } \\
\text { between } 0.05- \\
0.2 \mathrm{Vs}^{-1}\end{array}$ & $\begin{array}{l}\text { Proportional } \\
\text { LOD1 } \mu \mathrm{gL}^{-1}\end{array}$ & independent \\
\hline
\end{tabular}

Table 2. Analysis of carbamazepine in tablets and human plasma by DPV

\begin{tabular}{|l|c|c|c|c|}
\hline Sample & $\begin{array}{c}\text { Average } \\
(\mathrm{n}=3)\end{array}$ & SD & RSD (\%) & \% recovery \\
\hline Tablet 1 & $208.2 \mathrm{mg}$ & $4.3 \mathrm{mg}$ & 2.06 & 104.1 \\
\hline Tablet 2 & $201.3 \mathrm{mg}$ & $2.2 \mathrm{mg}$ & 1.09 & 100.6 \\
\hline Tablet 3 & $198.7 \mathrm{mg}$ & $3.1 \mathrm{mg}$ & 1.56 & 99.3 \\
\hline Tablet 4 & $192.0 \mathrm{mg}$ & $4.5 \mathrm{mg}$ & 2.34 & 96.0 \\
\hline Plasma 1 & $10.50 \mu \mathrm{g} \mathrm{mL}^{-1}$ & $0.95 \mu \mathrm{gL}^{-1}$ & 9.04 & 105 \\
\hline Plasma 2 & $9.80 \mu \mathrm{gL}^{-1}$ & $0.74 \mu \mathrm{gL}^{-1}$ & 7.55 & 98.0 \\
\hline Plasma 3 & $10.32 \mu \mathrm{g} \mathrm{mL}^{-1}$ & $0.56 \mu \mathrm{gLL}^{-1}$ & 5.42 & 103.2 \\
\hline Plasma 4 & $10.40 \mu \mathrm{g} \mathrm{mL}^{-1}$ & $0.61 \mu \mathrm{gL}^{-1}$ & 5.86 & 104.0 \\
\hline
\end{tabular}

